\title{
A VARIATIONAL APPROACH TO SPLINES
}

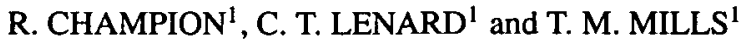

(Received 16 March 1998)

\begin{abstract}
This is an expository paper in which we present an introduction to a variational approach to spline interpolation. We present a sequence of theorems which starts with Holladay's classical result concerning natural cubic splines and culminates in some general abstract results.
\end{abstract}

\section{Introduction}

It is just over fifty years since Schoenberg [45] introduced "spline functions" or "splines" to the mathematical literature. Since then, splines have proved to be enormously popular in branches of mathematics such as approximation theory, numerical analysis and statistics. Also, they have become useful tools in fields of applications, especially computer-aided methods in manufacturing, in animation, in tomographyeven in surgery! We have now reached a point at which we can look back and trace the development of various trains of thought in the subject.

The aim of this expository paper is to draw attention to a variational approach to spline functions. We will see how a beautiful theory has evolved from a simple beginning. Also, we will observe that this theory exploits the power of functional analysis to give a clearer understanding of the essential features of a computational problem, namely, the problem of interpolation.

We wish to draw attention to this approach because, although the ideas are well known by experts in the field, we believe that the topic ought to be appreciated by a much wider audience of applied mathematicians, engineers and scientists who encounter splines in their work. As we will show, the variational approach gives a new way of thinking about splines and opens up avenues for theoretical developments and new applications. Despite this, the topic is not mentioned in many relevant texts on

\footnotetext{
${ }^{1}$ Division of Mathematics, La Trobe University, PO Box 199, Bendigo 3552, Australia.

(C) Australian Mathematical Society 2000, Serial-fee code 0334-2700/00
} 
numerical analysis or approximation theory: even books on splines tend to mention the variational approach only in passing or not at all. We hope that, in a small way, this paper addresses the lack of exposition of this important topic.

In Section 2, we introduce some notation and the usual definition of a spline. Then in Sections 3, 4 and 5 we sketch the development of a variational approach to splines. We do this (as one may do in a lecture) by means of a sequence of theorems of increasing generality or complexity which culminates in some very general but elegant theorems. The ideas are presented in an order which is not necessarily chronological: indeed, some of the very general ideas were known quite early in the piece. Rather, the order of presentation is designed to illuminate the most general ideas. A feature of our presentation is that we have tried to state theorems in a certain standard format in order to facilitate comparisons. In Section 6, we draw some conclusions from our considerations and make some comments on the literature.

\section{Preliminaries}

We will need some notation. In this paper, we let $\mathbf{R}$ denote the set of real numbers and $I$ denote the compact interval $[a, b] \subset \mathbf{R}$. The set of real polynomials of degree $m$ or less will be denoted by $\Pi_{m}$. A polynomial spline is usually defined as follows.

DEFINITION 1. Let $a=t_{0}<t_{1}<\cdots<t_{n}<t_{n+1}=b$ be a partition of $I$. Then $s: I \rightarrow \mathbf{R}$ is a polynomial spline of degree $m$ or less with respect to this partition if

- $s \in C^{m-1}(I)$ and

- for each $i \in\{0,1, \ldots, n\},\left.s\right|_{\left[t_{i}, t_{i+1}\right]} \in \Pi_{m}$.

The interior points $\left\{t_{1}, t_{2}, \ldots, t_{n}\right\}$ are known as "knots".

For any natural number $m, H^{m}(I)$ will denote the set of functions $x: I \rightarrow \mathbf{R}$ such that $x^{(m-1)}$ is absolutely continuous on $I$ and $x^{(m)} \in L^{2}(I)$. If we define an inner product on $H^{m}(I)$ by

$$
\left(x_{1}, x_{2}\right)=\int_{1} \sum_{j=0}^{m} x_{1}^{(j)}(t) x_{2}^{(j)}(t) d t
$$

then $H^{m}(I)$ becomes a Hilbert space.

If $X$ is a vector space, then $\theta_{X}$ will denote the zero element of $X$.

\section{Natural cubic splines}

We will be concerned with the problem of interpolation which can be stated as follows. Suppose that $t_{1}<t_{2}<\cdots<t_{n}$ and $\left\{z_{1}, z_{2}, \ldots, z_{n}\right\} \subset \mathbf{R}$. The problem 
of interpolation is to find a "nice" function $\phi$ which interpolates the data points $\left(t_{i}, z_{i}\right)(1 \leq i \leq n)$ : that is,

$$
\phi\left(t_{i}\right)=z_{i} \quad(1 \leq i \leq n) .
$$

Classical approaches developed by Lagrange, Hermite, Cauchy and others rely on choosing $\phi$ to be some suitable polynomial. To be sure, polynomials are nice functions from almost any point of view and they are often used effectively for interpolation. But are there better functions for solving this interpolation problem? One approach to this question can be found in a result which was proved by Holladay [29] in 1957.

THEOREM 1 (Holladay). If

- $X=H^{2}(I)$

- $a \leq t_{1}<t_{2}<\cdots<t_{n} \leq b ; n \geq 2$,

- $\left\{z_{1}, z_{2}, \ldots, z_{n}\right\} \subset \mathbf{R}$ and

- $I_{n}=\left\{x \in X: x\left(t_{i}\right)=z_{i} \quad(1 \leq i \leq n)\right\}$,

then $\exists ! \sigma \in I_{n}$ :

$$
\int_{I} \sigma^{(2)}(t)^{2} d t=\min \left\{\int_{I} x^{(2)}(t)^{2} d t: x \in I_{n}\right\}
$$

Furthermore,

- $\sigma \in C^{2}(I)$,

- $1 \leq i \leq n-\left.1 \Rightarrow \sigma\right|_{\left[t_{i}, i_{i+1}\right]} \in \Pi_{3}$ and

- $\left.\sigma\right|_{\left[a, \ell_{1}\right]} \in \Pi_{1}$ and $\left.\sigma\right|_{\left[t_{n}, b\right]} \in \Pi_{1}$.

From (1), we see that $\sigma$ is an optimal interpolating function- "optimal" in the sense that it minimizes $\int_{I} x^{(2)}(t)^{2} d t$ over all functions in $I_{n}$. The theorem goes on to state that $\sigma$ is a cubic spline. As $\sigma$ is linear outside $\left[t_{1}, t_{n}\right]$, it is called a "natural" cubic spline.

Since $\sigma$ is an optimal interpolating function, we can say that the natural cubic interpolating spline is better than any interpolating polynomial (which would certainly be a member of $I_{n}$ ). So, in a technical sense, we have found functions which are better than polynomials for solving the interpolation problem.

Holladay's theorem is most surprising. As the set $I_{n}$ contains a vast array of functions, the fact that the form of the optimal function is so simple is amazing. Still more pleasing is the fact that the proof is quite elementary, relying on nothing more complicated than integration by parts. (See for example, Ahlberg, Nilson and Walsh $[3$, p. 76].)

So, cubic splines provide us with optimal interpolating functions. It is this intrinsic aspect of splines as solutions to variational problems like (1) that mathematicians have exploited to develop a variational approach to splines. 
It is natural to ask "Why would one choose to minimize $\int_{I} x^{(2)}(t)^{2} d t$ "? Holladay gives us a physical interpretation of the significance of this objective function, but here is another (which was suggested by Schoenberg [46]).

When presented with a set of data points $\left(t_{i}, z_{i}\right)(1 \leq i \leq n)$, a statistician can find a regression line which is the line of best fit in the least squares sense. This line is close to the data points.

Now if $x$ were a linear function, then we would have $\int_{I} x^{(2)}(t)^{2} d t=0$; but generally speaking, we cannot find a linear function which interpolates the data. Holladay's theorem shows that $\sigma$ minimizes $\int_{1} x^{(2)}(t)^{2} d t$ while still interpolating the data. We could say that $\sigma$ is an interpolating function which is "close to a straight line" in that it minimizes this integral.

Thus, linear regression gives us

$$
\text { a straight line passing close to the points }
$$

whereas Holladay's result gives a curve which is

close to a straight line but passing through the points.

As we will see in the next section, Holladay's simple result has become the starting point for the development of a beautiful theory.

\section{More splines}

In this section, we describe a few of the many generalisations and extensions of Holladay's theorem.

\section{$D^{m}$-splines}

The next step was taken in 1963 by Carl de Boor [13] with the following result.

THEOREM 2 (de Boor). If

- $X=H^{m}(I)$,

- $a \leq t_{1}<t_{2}<\cdots<t_{n} \leq b ; n \geq m$,

- $\left\{z_{1}, z_{2}, \ldots, z_{n}\right\} \subset \mathbf{R}$ and

- $I_{n}=\left\{x \in X: x\left(t_{i}\right)=z_{i} \quad(1 \leq i \leq n)\right\}$,

then $\exists ! \sigma \in I_{n}$ :

$$
\int_{I} \sigma^{(m)}(t)^{2} d t=\min \left\{\int_{I} x^{(m)}(t)^{2} d t: x \in I_{n}\right\}
$$

Furthermore, 
- $\sigma \in C^{2 m-2}(I)$,

- $1 \leq i \leq n-\left.1 \Rightarrow \sigma\right|_{\left[t_{i}, i_{i+1}\right]} \in \Pi_{2 m-1}$ and

- $\left.\sigma\right|_{\left[a, t_{1}\right]} \in \Pi_{m-1}$ and $\left.\sigma\right|_{\left[t_{n}, b\right]} \in \Pi_{m-1}$.

Today, some would describe $\sigma$ as a $D^{m}$-spline because it minimizes $\int_{I}\left(D^{m} x\right)^{2}$ as $x$ varies over $I_{n}$. (Here, as elsewhere, $D^{m} x$ denotes the $m$-th derivative of $x$ with respect to its argument.) Clearly, if we let $m=2$ in de Boor's result, then we obtain Holladay's result.

\section{Trigonometric splines}

In 1964, Schoenberg [46] changed the setting of the interpolation problem from the interval $[a, b]$ to the unit circle: that is, from a non-periodic setting to a periodic setting. Accordingly, we let $H_{2 \pi}^{k}([0,2 \pi))$ denote the space of $2 \pi$-periodic functions $x:[0,2 \pi) \rightarrow \mathbf{R}$ such that $x^{(k-1)}$ is absolutely continuous on $[0,2 \pi)$ and $x^{(k)} \in$ $L_{2 \pi}^{2}([0,2 \pi))$. Schoenberg's result can be stated as follows.

THEOREM 3 (Schoenberg). If

- $X=H_{2 \pi}^{2 m+1}([0,2 \pi))$,

- $0 \leq t_{1}<t_{2}<\cdots<t_{n}<2 \pi ; n>2 m+1$,

- $\left\{z_{1}, z_{2}, \ldots, z_{n}\right\} \subset \mathbf{R}$,

- $I_{n}=\left\{x \in X: x\left(t_{i}\right)=z_{i} \quad(1 \leq i \leq n)\right\}$ and

- $T: X \rightarrow L_{2 \pi}^{2}([0,2 \pi))$, where $T=D\left(D^{2}+1^{2}\right) \cdots\left(D^{2}+m^{2}\right)$,

then $\exists ! \sigma \in I_{n}$ :

$$
\int_{0}^{2 \pi} T[\sigma](t)^{2} d t=\min \left\{\int_{0}^{2 \pi} T[x](t)^{2} d t: x \in I_{n}\right\} .
$$

The optimal interpolating function $\sigma$ is a trigonometric spline. Schoenberg defines a trigonometric spline as a smooth function which is constructed in a particular piecewise manner. He shows that trigonometric splines, so defined, provide the solution of this problem.

Note that the differential operator $T$ will annihilate $x$ if $x$ is a trigonometric polynomial of order $m$, that is, of the form

$$
x(t)=a_{0}+\sum_{j=1}^{m}\left(a_{j} \cos j t+b_{j} \sin j t\right) .
$$

\section{$g$-Splines}

Just over 200 years ago, Lagrange [35] showed us how to construct a polynomial of minimal degree whose graph passed through prescribed data points: that is, the 
polynomial assumed prescribed values at given points. (For interesting historical remarks on Lagrange interpolation, see Elliott [19].) In 1878, Hermite [28] made an important contribution to the development of interpolation methods. He showed us how to construct a polynomial of minimal degree such that (i) the polynomial assumed prescribed values at given nodes, and (ii) the derivatives of certain orders of the polynomial also assumed prescribed values at the nodes.

In our discussion of splines, we have, up to this point, considered only splines whose values are prescribed at given points: that is, we have a Lagrange type of problem. Could we extend these results using Hermite's idea? In 1968, Schoenberg [47] showed us how to effect such an extension.

To state Schoenberg's result we need a little machinery. As usual we will have $n$ nodes $t_{1}<t_{2}<\cdots<t_{n}$ in $I=[a, b]$, but we will want to specify the value of the spline and some of its derivatives at the nodes. Now the actual orders of the derivatives specified may vary from node to node. To help indicate this, we introduce an incidence matrix $E$.

Suppose that, as usual, $a \leq t_{1}<t_{2}<\cdots t_{n} \leq b$. Let $\ell$ be the maximum of the orders of the derivatives to be specified at the nodes. We will assume that

$$
E=(e(i, j): 1 \leq i \leq n, 0 \leq j \leq \ell)=(e(i, j)),
$$

where each $e(i, j)$ is 0 or 1 . Assume also that each row of $E$ and the last column of $E$ contain a 1.

DEFINITION 2. If $m \geq 1$ is an integer, we will say that the incidence matrix $E=$ $(e(i, j))$ is $m$-poised with respect to $t_{1}<t_{2}<\cdots<t_{n}$ if

- $P \in \Pi_{m-1}$ and

- $e(i, j)=1 \Rightarrow P^{(j)}\left(t_{i}\right)=0$

together imply that $P \equiv 0$.

Now we can state Schoenberg's result.

THEOREM 4 (Schoenberg). If

- $X=H^{m}(I)$,

- $a \leq t_{1}<t_{2}<\cdots<t_{n} \leq b$,

- $E$ is an m-poised incidence matrix of dimensions $n \times(\ell+1)$,

- $\ell<m \leq \sum_{i} \sum_{j} e(i, j)$,

- $\left\{z_{i j}: e(i, j)=1\right\} \subset \mathbf{R}$ and

- $I_{n}=\left\{x \in X: x^{(j)}\left(t_{i}\right)=z_{i j}\right.$ if $\left.e(i, j)=1\right\}$,

then $\exists ! \sigma \in I_{n}$ :

$$
\int_{I} \sigma^{(m)}(t)^{2} d t=\min \left\{\int_{I} x^{(m)}(t)^{2} d t: x \in I_{n}\right\}
$$


The optimal function $\sigma$ is known as a " $g$-spline". It seems that Schoenberg would have liked to use the term "generalized spline" for $\sigma$, but this had been coined by Ahlberg and Nilson for another purpose. So he settled for $g$-spline (see [47, p. 208]). In retrospect, naming them $H$-splines after Hermite or $H B$-splines after Hermite and Birkhoff may have been appropriate.

Again, Schoenberg defines $g$-splines as smooth, piecewise polynomials where the smoothness is governed by $E$ and then he proves that $g$-splines solve the above variational problem.

\section{$L$-Splines}

In an important paper published in 1967, Schultz and Varga [48] discuss a major extension of the $D^{m}$-spline. To give the flavour of this extension we state only one, simple consequence of the many results in this paper.

THEOREM 5. If

- $X=H^{m}(I)$,

- $a \leq t_{1}<t_{2}<\cdots<t_{n} \leq b ; n \geq m$,

- $\left\{z_{1}, z_{2}, \ldots, z_{n}\right\} \subset \mathbf{R}$,

- $I_{n}=\left\{x \in X: x\left(t_{i}\right)=z_{i}(1 \leq i \leq n)\right\}$,

- $L: X \rightarrow L^{2}(I)$ so that $L[x](t)=\sum_{j=0}^{m} a_{j}(t) D^{j} x(t)$ where $a_{j} \in C^{j}(I)(0 \leq$ $j \leq m)$ and $\exists w>0$ such that $a_{m}(t) \geq w>0$ on $I$ and

- L has Pólya's property W on I,

then $\exists ! \sigma \in I_{n}$ :

$$
\int_{I} L[\sigma](t)^{2} d t=\min \left\{\int_{I} L[x](t)^{2} d t: x \in I_{n}\right\} .
$$

Clearly complexity is increasing with generality! For completeness, we note that $L$ has Pólya's property $W$ on $I$ if $L[x]=0$ has $m$ solutions $x_{1}, x_{2}, \ldots, x_{m}$ such that, for all $t \in I$ and for all $k \in\{1,2, \ldots, m\}$,

$$
\operatorname{det}\left[\begin{array}{cccc}
x_{1}(t) & x_{2}(t) & \cdots & x_{k}(t) \\
D x_{1}(t) & D x_{2}(t) & \cdots & D x_{k}(t) \\
\cdot & \cdot & \cdots & \cdot \\
\cdot & \cdot & \cdots & \cdot \\
\cdot & \cdot & \cdots & \cdot \\
D^{k-1} x_{1}(t) & D^{k-1} x_{2}(t) & \cdots & D^{k-1} x_{k}(t)
\end{array}\right] \neq 0 .
$$

The relevance of Pólya's property $W$ is contained in the following sentence. To say that $L$ has Pólya's property $W$ on $I$ implies that, if (i) $L[x]=0$ and (ii) $x$ has $m$ or more zeros on $I$, then $x \equiv 0$ (see [27]). 
The optimal function $\sigma$ is known as an $L$-spline. If $L=D^{m}$ then we obtain the $D^{m}$-spline; so this is a major extension of previously stated results.

Schultz and Varga define an $L$-spline to be a smooth function constructed in a piecewise manner, where each piece is a solution of the differential equation $L^{*} L x=0$ where $L^{*}$ is the formal adjoint of the operator $L$. A consequence of their paper is that $L$-splines provide the solution of the above variational problem.

As said earlier, the above result is only one consequence of the paper of Schultz and Varga. In fact this result was proved in 1964 by Ahlberg, Nilson and Walsh [2]: they call $\sigma$ a "generalized spline". (Schultz and Varga chose the better name.) The work of Schultz and Varga allows more complex interpolation conditions. The above result also follows from the paper by de Boor and Lynch [15]. Perhaps the first paper along these lines of replacing the operator $D^{m}$ by a more general differential operator was by Greville [25] also in 1964. Unfortunately this often cited technical report was never published.

Greville illustrates his methods with an application to the classical numerical problem of interpolating mortality tables. Schultz and Varga apply their ideas to the numerical analysis of nonlinear two-point boundary value problems. Prenter [42] is one of the few text books which touches this topic.

\section{Lg-Splines}

Schoenberg extended the concept of $D^{m}$-splines to allow interpolation conditions of the Hermite type: this led to $g$-splines. Schultz and Varga (and others) extended the concept of a $D^{m}$-spline in a different direction by replacing the differential operator $D^{m}$ by a more general operator: this led to $L$-splines. Now Schultz and Varga ([48, Section 5]) noticed that one could combine both these extensions. But it was a paper by Jerome and Schumaker [33] in 1969 which moulded these two extensions together in a very effective manner. We now state one of their results.

THEOREM 6 (Jerome and Schumaker). If

- $X=H^{m}(I)$,

- $\left\{\lambda_{1}, \lambda_{2}, \ldots, \lambda_{n}\right\}$ is a set of linearly independent, continuous linear functionals on $X$,

- $\left\{z_{1}, z_{2}, \ldots, z_{n}\right\} \subset \mathbf{R}$,

- $I_{n}=\left\{x \in X: \lambda_{i}(x)=z_{i}(1 \leq i \leq n)\right\}$,

- $L: X \rightarrow L^{2}(I)$ so that $L[x](t)=\sum_{j=0}^{m} a_{j}(t) D^{j} x(t)$, where $a_{j} \in C^{j}(I)$ $(0 \leq j \leq m)$ and $\exists w>0$ such that $a_{m}(t) \geq w>0$ on $l$ and

- $\operatorname{ker} L \cap\left\{x \in X: \lambda_{i}(x)=0(1 \leq i \leq n)\right\}=\left\{\theta_{X}\right\}$, 
then $\exists ! \sigma \in I_{n}$ :

$$
\int_{I} L[\sigma](t)^{2} d t=\min \left\{\int_{I} L[x](t)^{2} d t: x \in I_{n}\right\}
$$

The optimal function $\sigma$ is called an $L g$-spline. Jerome and Schumaker define an $L g$-spline to be the solution of the variational interpolation problem. As we have seen, previous writers had defined various types of generalised splines as smooth functions constructed in some piecewise manner and then proved that these generalised splines provided the solution of some variational problem. Note also that the hypothesis about Pólya's property $W$ in Theorem 5 has been replaced by an hypothesis with a more functional-analytic flavour.

In addition to combining the notion of an $L$-spline and the notion of a $g$-spline, Jerome and Schumaker have also extended the notion of a $g$-spline. Schoenberg's interpolating $g$-spline was a function with prescribed values at the nodes and with prescribed derivatives of certain orders at the nodes. However, Jerome and Schumaker allow interpolation conditions of the more general form $\lambda_{i}(x)=z_{i}(1 \leq i \leq n)$ where the $\lambda_{i}(i=1,2, \ldots, n)$ are continuous linear functionals on $X$. This idea could cover not only Schoenberg's type of interpolation conditions but also others (e.g. $\left.\int_{t_{i}}^{t_{i+1}} x(t) d t=z_{i}\right)$.

We note also that in Section 7 of their paper, Jerome and Schumaker extend the interpolation conditions in the variational problem even further. They replace the conditions $\lambda_{i}(x)=z_{i}(1 \leq i \leq n)$ by $\underline{z}_{i} \leq \lambda_{i}(x) \leq \bar{z}_{i}(1 \leq i \leq n)$, where $\left\{\underline{z}_{i}: i=1,2, \ldots, n\right\} \subset \mathbf{R},\left\{\bar{z}_{i}: i=1,2, \ldots, n\right\} \subset \mathbf{R}$ and $\underline{z}_{i} \leq \bar{z}_{i}(i=1,2, \ldots, n)$.

Discussion of $L$-splines and $L g$-splines can be found in [30, 34] and [53].

\section{$p L g-$ Splines}

If $1<p<\infty$ then we define $H^{m, p}(I)$ to be the space of functions $x: I \rightarrow \mathbf{R}$ such that $x^{(m-1)}$ is absolutely continuous on $I$ and $x^{(m)} \in L^{p}(I)$. We can define a norm on $H^{m, p}(I)$ by

$$
\|x\|_{m, p}=\sum_{j=0}^{m-1}\left|x^{(j)}(a)\right|+\left(\int_{I}\left|x^{(m)}(t)\right|^{p} d t\right)^{1 / p}
$$

We can now state a result from Copley and Schumaker [12] which was published in 1978.

THEOREM 7 (Copley and Schumaker). If

- $X=H^{m, p}(I), p>1$, 
- $\left\{\lambda_{1}, \lambda_{2}, \ldots, \lambda_{n}\right\}$ is a set of linearly independent continuous linear functionals on $X$,

- $\left\{z_{1}, z_{2}, \ldots, z_{n}\right\} \subset \mathbf{R}$,

- $I_{n}=\left\{x \in X: \lambda_{i}(x)=z_{i}(1 \leq i \leq n)\right\} \neq \emptyset$,

- $L: X \rightarrow L^{p}(I)$ so that $L[x](t)=\sum_{j=0}^{m} a_{j}(t) D^{j} x(t)$ where $a_{j} \in C^{j}(I)$ $(0 \leq j \leq m)$ and $\exists w>0$ such that $a_{m}(t) \geq w>0$ on $I$ and

- $\operatorname{ker} L \cap\left\{x \in X: \lambda_{i}(x)=0(1 \leq i \leq n)\right\}=\left\{\theta_{X}\right\}$,

then $\exists$ ! $\sigma \in I_{n}$ :

$$
\int_{I}|L[\sigma](t)|^{p} d t=\min \left\{\int_{I}|L[x](t)|^{p} d t: x \in I_{n}\right\} .
$$

The optimal function $\sigma$ is called a $p L g$-spline. Copley and Schumaker define a $p L g$-spline to be a solution of the variational interpolation problem. One of the main problems that they investigate is to determine the structure of such splines. Can they be constructed in a piecewise manner? The complexity of their answers highlights the simplicity of their definition of a $p L g$-spline.

The above theorem is intended only to give the flavour of [12]: in fact Copley and Schumaker investigate more general interpolation problems than those in the above theorem. For example they consider sets of linear functionals $\left\{\lambda_{\alpha}: \alpha \in A\right\}$, where the index set $A$ may be infinite. One of the nice features of this paper is the section on worked examples.

\section{Vector-valued $L g$-splines}

Other extensions have come from researchers in electrical engineering: Sidhu and Weinert [49] presented one such extension in 1979. They consider the problem of simultaneous interpolation, that is, a method by which one could interpolate several functions at once.

THEOREM 8 (Sidhu and Weinert). If

- $r \geq 1, n_{1} \geq 0, \ldots, n_{r} \geq 0$ are fixed integers,

- $X=H^{n_{1}}(I) \times \ldots \times H^{n_{r}}(I)$,

- $\left\{\lambda_{1}, \lambda_{2}, \ldots, \lambda_{n}\right\}$ is a set of linearly independent continuous linear functionals on $X$,

- $\left\{z_{1}, z_{2}, \ldots, z_{n}\right\} \subset \mathbf{R}$,

- $I_{n}=\left\{x \in X: \lambda_{i}(x)=z_{i}(1 \leq i \leq n)\right\}$,

- $L: X \rightarrow L^{2}(I) \times \cdots \times L^{2}(I)$ (an r-fold product), where

$$
L[x](t)=\left(\sum_{j=1}^{r} L_{i j}\left[x_{j}\right](t): i=1,2, \ldots, r\right)^{\prime},
$$




$$
L_{i j}=\sum_{k=0}^{n_{j}} a_{i j k}(t) D^{k} ; \quad a_{i j n_{j}}=\delta_{i j} ; a_{i j k} \in C^{k}(I)\left(0 \leq k \leq n_{j}\right) \quad \text { and }
$$

- $\operatorname{ker} L \cap\left\{x \in X: \lambda_{i}(x)=0(1 \leq i \leq n)\right\}=\left\{\theta_{X}\right\}$,

then $\exists ! \sigma \in X$ :

$$
\int_{I}(L[\sigma](t))^{\prime} L[\sigma](t) d t=\min \left\{\int_{I}(L[x](t))^{\prime} L[x](t) d t: x \in I_{n}\right\} .
$$

(Here $A^{\prime}$ indicates the transpose of the matrix or vector $A$.)

The optimal interpolating vector $\sigma$ is known as a vector-valued $L g$-spline. The authors define a vector-valued $L g$-spline to be the solution of a variational interpolation problem, prove the above existence-uniqueness theorem and then discuss an algorithm for calculating such splines in the special case that the functionals $\lambda_{i}$ are of extended Hermite-Birkhoff type.

\section{Thin plate splines}

So far we have been considering the problem of interpolating functions of a single variable. In 1976, Jean Duchon [18] proved a result which is regarded as a significant step towards developing a variational approach to interpolating functions of several variables. Below we state his result for functions of two variables because it is relatively simple to state and quite dramatic. We will denote an arbitrary element of $\mathbf{R}^{2}$ by $t=\left(\xi_{1}, \xi_{2}\right),\|t\|^{2}:=\xi_{1}^{2}+\xi_{2}^{2}$, and the set of linear polynomials by

$$
\Pi_{1}:=\left\{p_{1}(t)=a_{0}+a_{1} \xi_{1}+a_{2} \xi_{2}:\left\{a_{0}, a_{1}, a_{2}\right\} \subset \mathbf{R}\right\}
$$

THEOREM 9 (Duchon). If

- $X=H^{2}\left(\mathbf{R}^{2}\right)$,

- $\left\{t_{1}, t_{2}, \ldots, t_{n}\right\} \subset \mathbf{R}^{2}$ such that if $p_{1} \in \Pi_{1}$ and $p_{1}\left(t_{1}\right)=\cdots=p_{1}\left(t_{n}\right)=0$ then $p_{1} \equiv 0$,

- $\left\{z_{1}, z_{2}, \ldots, z_{n}\right\} \subset \mathbf{R}$,

- $I_{n}=\left\{x \in X: x\left(t_{i}\right)=z_{i}(1 \leq i \leq n)\right\}$ and

- $J: X \rightarrow \mathbf{R}$ such that

$$
J(x)=\iint_{\mathbf{R}^{2}}\left(\frac{\partial^{2} x}{\partial \xi_{1}^{2}}\right)^{2}+2\left(\frac{\partial^{2} x}{\partial \xi_{1} \partial \xi_{2}}\right)^{2}+\left(\frac{\partial^{2} x}{\partial \xi_{2}^{2}}\right)^{2} d \xi_{1} \xi_{2},
$$

then $\exists ! \sigma \in I_{n}$ :

$$
J(\sigma)=\min \left\{J(x): x \in I_{n}\right\}
$$


Furthermore, $\forall t \in \mathbf{R}^{2}$,

$$
\sigma(t)=\sum_{j=1}^{n} \lambda_{i}\left\|t-t_{i}\right\|^{2} \ln \left\|t-t_{i}\right\|+p_{1}(t),
$$

where

- $p_{1} \in \Pi_{1}$ and

- $\left(\forall q \in \Pi_{1}\right)\left(\sum_{i=1}^{n} \lambda_{i} q\left(t_{i}\right)=0\right)$.

The optimal function $\sigma$ is known as a "thin plate spline". The dramatic aspect of this result is the form of the spline $\sigma$ : it is nothing like a piecewise polynomial function.

This two-dimensional result appeared almost 20 years after Holladay's one-dimensional result. Given the difference in techniques used to prove these results, the delay is not so surprising. Holladay's proof involves nothing more complicated than integration by parts whereas Duchon's paper uses tempered distributions, Radon measures and other tools from functional analysis. A more elementary approach to Duchon's result is outlined in Powell [41].

Although the present paper is not intended to be a complete survey of the literature, it is appropriate to point out that Duchon was not the first person to tackle the multivariate problem. Duchon notes the work of two aircraft engineers Harder and Desmaris [26] who approached this problem from an applied point of view. In 1974, Fisher and Jerome [20] addressed the multivariate problem. In his doctoral thesis in 1970, Jean Thomann [51] considered a variational approach to interpolation on a rectangle or on a disk in $\mathbf{R}^{2}$. In any major review of thin plate splines, these three publications deserve attention. The book by Ahlberg, Nilson and Walsh [3] also deals with multivariate problems, but from a point of view which is essentially univariate.

\section{Yet more splines}

The litany of splines could be continued. There are $\Lambda$-splines [32], $L M g$-splines [16], $A R M A$-splines [56], $P D L g$-splines [17], spherical splines [54, 22], hyperspherical splines [50], vector splines [4, 5], and polyharmonic splines [43]. These too are splines associated with some variational interpolation problem. In each case we could state a theorem similar in flavour to those above-but, alas, space does not permit this.

\section{Abstract splines}

The statements of the theorems above were becoming quite long and complicated. Indeed, there is a general abstract result which captures the essence of most of them. 
We attribute the following result to $M$. Atteia and it relates to the following diagram.

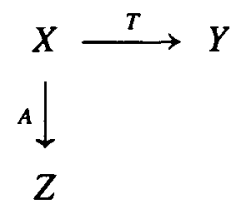

THEOREM 10 (Atteia). If

- $X, Y, Z$ are Hilbert spaces,

- $z \in Z$,

- $T, A$ are continuous, linear surjections,

- $\operatorname{ker} T+\operatorname{ker} A$ is closed in $X$,

- $\operatorname{ker} T \cap \operatorname{ker} A=\left\{\theta_{X}\right\}$ and

- $l(z)=\{x \in X: A x=z\}$,

then $\exists ! \sigma \in I(z)$ :

$$
\|T \sigma\|_{Y}=\min \left\{\|T x\|_{Y}: x \in I(z)\right\} .
$$

The optimal $\sigma$ is known as a variational interpolating spline. To illustrate that this theorem reflects the essence of some results above, let us see how it generalises Theorem 1 of Holladay. Put $X=H^{2}(I), Y=L^{2}(I), Z=\mathbf{R}^{n}, T(x):=x^{(2)}, A(x):=$ $\left(x\left(t_{1}\right), x\left(t_{2}\right), \ldots, x\left(t_{n}\right)\right)$, and then check that the hypotheses of Atteia's theorem are satisfied. Atteia's theorem does not cover all the above results (e.g. Theorem 7 which deals with $p L g$-splines).

We have attributed the above result to Atteia because, as far as we can tell, the ideas grew out of his 1965 paper [7] and his thesis [8]. An equivalent result is found in the often cited, but unfortunately never published, report by Golomb [23]. The essential ideas also can be found in Anselone and Laurent [6] and the classic work by Laurent [36]. The theorem, as stated above, can be found in Atteia [9].

There are three observations that we would like to make about this theorem.

First, the role of the condition about $\operatorname{ker} T+\operatorname{ker} A$ is to ensure the existence of $\sigma$ whereas the role of the condition about $\operatorname{ker} T \cap \operatorname{ker} A$ is to ensure the uniqueness of $\sigma$. This separation was made clear in Jerome and Schumaker [33].

Second, the above theorem is only an existence-uniqueness theorem. The challenge for any abstract theory is to generalise a wide variety of particular cases, and simultaneously, preserve as much of the detail as possible. To a large extent, Atteia and others have, over many years, being doing this in the case that $X$ is a reproducing kernel Hilbert space. Details of this program can be found in [9] and [11]. The origins of this program can be found in a 1959 paper by Golomb and Weinberger [24], Atteia [8] and de Boor and Lynch [15].

Third, the above general theorem can itself be generalised in many directions. 
One generalisation enables us to consider constrained interpolation problems which are very important in contemporary numerical mathematics. It is due to Utreras [52] and relates to the following diagram.

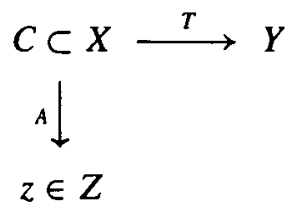

THEOREM 11 (Utreras). If

- $X, Y, Z$ are Hilbert spaces,

- $C$ is a closed, convex subset of $X$,

- $z \in Z$,

- $A, T$ are continuous, linear surjections,

- $w \in I(C, z):=\{x \in C: A x=z\}$,

- $\operatorname{ker} T+(\operatorname{ker} A \cap(C-w))$ is closed in $X$ and

- $\operatorname{ker} A \cap \operatorname{ker} T=\left\{\theta_{X}\right\}$,

then $\exists ! \sigma \in I(C, z)$ :

$$
\|T \sigma\|_{Y}=\min \left\{\|T x\|_{Y}: x \in I(C, z)\right\} .
$$

If we put $C=X$ then we obtain Theorem 10. Utreras' theorem is useful if, for example, we want to interpolate positive data by positive functions. In this case, we may have $X=H^{m}(I)$ and $C$ being the set of positive functions in $X$.

Other generalisations have extended Atteia's theorem to Banach space settings rather than Hilbert space settings. Holmes [31] writes about $R$-splines, Pai [40] writes about $L f$-splines, and Benbourhim and Gaches [10] write about $T_{f}$-splines. A key work in the Banach space setting is Fisher and Jerome [21]. Perfect splines are important in this context.

Lucas $[38,39]$ took abstract splines in quite a different direction with his notion of an $M$-spline. See also $[1,44]$.

In short, a beautiful mathematical theory of variational interpolating splines has developed from the elementary theorem of Holladay.

\section{Conclusions}

We have presented an introduction to a variational theory of interpolating splines in order to draw attention to this approach. This exposition may be helpful as an introduction to the few books which deal with these ideas. The book by Laurent [36] was perhaps the first book which emphasised the variational approach to splines. Atteia's 
book [9] is a key work in this area for those interested in functional analysis. Wahba [55] describes applications of these ideas (in smoothing rather than interpolation) to statistics. Bezhaev and Vasilenko [11] is a recent book in the field but difficult to obtain.

There are three conclusions that we would like to draw from the above exposition.

1. Splines may be defined as solutions of variational problems rather than functions constructed in some piecewise manner. We have seen that, initially, splines were defined as smooth functions which could be constructed in some piecewise manner. Eventually, it became convenient to define an interpolating spline as the solution of some variational problem. We have also seen that these variational problems have become increasingly abstract and hence the very concept of a "spline" has become increasingly abstract. This may not be to everyone's liking. For example, in [15], de Boor and Lynch write "in order not to dilute the notion of spline functions too much, we prefer to follow Greville's definition of a general spline function"-which is based on a piecewise, constructive approach. Perhaps the point at which one gets off the ladder of increasing abstraction is a matter of personal taste. In any case, the variational theory gives us a new appreciation of the concept of a "spline".

2. The variational approach facilitates a natural, attractive way to extend the classical theory of interpolating splines, especially to multivariate situations. The works of Duchon [18] and of Wahba [54] in particular illustrate this conclusion. More recently de Boor [14] wrote "I am convinced that the variational approach to splines will play a much greater role in multivariate spline theory that it did or should have in univariate theory."

3. The theory of variational splines demonstrates the power of functional analysis to yield a unified approach to computational problems in interpolation. As S. Sobolev has been quoted as saying [37] "It is impossible to imagine the theory of computations with no Banach spaces".

\section{Acknowledgements}

T. M. Mills acknowledges support from (i) La Trobe University for an OSP grant to visit Université Paul Sabatier in Toulouse in late 1995 and (ii) both La Trobe University and The Technical University of Budapest to travel to Budapest in 1997. Both these visits helped him to gather material for this paper. The authors are very grateful to Professor R. S. Varga for helpful comments during the preparation of this paper.

\section{References}

[1] V. Abraham, "On the existence and uniqueness of $M$-splines", J. Approx. Theory 43 (1985) 36-42. 
[2] J. H. Ahlberg, E. N. Nilson and J. L. Walsh, "Fundamental properties of generalized splines", Proc. Nat. Acad. Sci. USA 52 (1964) 1412-1419.

[3] J. H. Ahlberg, E. N. Nilson and J. L. Walsh, The Theory of splines and Their Applications (Academic Press, New York, 1967).

[4] L. Amodei, "Étude d'une classe de fonctions splines vectorielles en vue de l'approximation d'un champ de vitesse. Applications à la météorologie", Thèse, Université Paul Sabatier, Toulouse, 1993.

[5] L. Amodei and M. N. Benbourhim, "A vector spline approximation", J. Approx. Theory 67 (1991) 51-79.

[6] P. M. Anselone and P. J. Laurent, "A general method for the construction of interpolating or smoothing spline-functions", Numer. Math. 12 (1968) 66-82.

[7] M. Atteia, "Généralisation de la définition et des propriétés des spline fonctions", C.R. Acad. Sc. Paris 260 (1965) 3550-3553.

[8] M. Atteia, "Étude de certains noyaux et théorie des fonctions spline en analyse numérique", Thèse, l'Instit. Math. Appl., Grenoble, 1966.

[9] M. Atteia, Hilbertian Kernels and Spline Functions (North Holland, Amsterdam, 1992).

[10] N. M. Benbourhim and J. Gaches, " $T_{f}$-splines et approximation par $T_{f}$-prolongement", Studia Math. 106 (1993) 203-211.

[11] A. Yu. Bezhaev and V. A. Vasilenko, Variational Spline Theory (Novosibirsk Computing Center, Novosibirsk, 1993).

[12] P. Copley and L. L. Schumaker, "On $p L g$-splines", J. Approx. Theory 23 (1978) 1-28.

[13] C. de Boor, "Best approximation properties of spline functions of odd degree", J. Math. Mech. 12 (1963) 747-749.

[14] C. de Boor, "Multivariate piecewise polynomials", Acta Numerica (1993) 65-109.

[15] C. de Boor and R. E. Lynch, "On splines and their minimum properties", J. Math. Mech. 15 (1966) 953-969.

[16] R. J. P. de Figueiredo, “LM-g splines”, J. Approx. Theory 19 (1979) 332-360.

[17] R. J. P. de Figueiredo and G. Chen, " $P D L_{g}$ splines defined by partial differential operators with initial and boundary value conditions", SIAM J. Numer. Anal. 27 (1990) 519-528.

[18] J. Duchon, "Interpolation des fonctions de deux variables suivant le principe de la flexion des plaques minces”, R.A.I.R.O. Analyse Numérique 10 (1976) 5-12.

[19] D. Elliott, "Lagrange interpolation-decline and fall?", Internat. J. Math. Ed. Sci. Tech. 10 (1979) 1-12.

[20] S. D. Fisher and J. W. Jerome, "Elliptic variational problems in $L^{2}$ and $L^{\infty}$ ", Indiana Univ. Math. Jnl. 23 (1974) 685-698.

[21] S. D. Fisher and J. W. Jerome, Minimum Norm Extremals in Function Spaces, Lecture Notes in Math. 479 (Springer, Berlin, 1975).

[22] W. Freeden, M. Schreiner and R. Franke, "A survey of spherical spline approximation", Surveys on Mathematics for Industry (to appear).

[23] M. Golomb, "Splines, $n$-widths and optimal approximations", MRC Technical Summary Report 784, Mathematics Research Center, US Army, Madison, Wisconsin, 1967.

[24] M. Golomb and H. F. Weinberger, "Optimal approximation and error bounds", in On Numerical Approximation (ed. R. E. Langer), (Univ. Wisconsin Press, Madison, 1959) 117-190.

[25] T. N. E. Greville, "Interpolation by generalized spline functions", MRC Technical Summary Report 476, Mathematics Research Center, US Army, Madison, Wisconsin, 1964.

[26] R. L. Harder and R. N. Desmarais, "Interpolation using surface splines", J. Aircraft 9 (1972) 189-191.

[27] P. Hartman, Ordinary Differential Equations, second ed. (Birkhaüser, Boston, 1982).

[28] Ch. Hermite, "Sur la formule d'interpolation de Lagrange", J. Reine Angew. Math. 84 (1878) 70-79. 
[29] J. C. Holladay, “A smoothest curve approximation", Math. Tables Aids Comput. 11 (1957) 233-243.

[30] A. S. B. Holland and B. N. Sahney, The General Problem of Approximation and Spline Functions (Robert E. Krieger Pub., Huntington, 1979).

[31] R. Holmes, " $R$-splines in Banach spaces: I. Interpolation of linear manifolds", J. Math. Anal. Appl. 40 (1972) 574-593.

[32] J. W. Jerome and J. Pierce, "On spline functions determined by singular self-adjoint differential operators", J. Approx. Theory 5 (1972) 15-40.

[33] J. W. Jerome and L. L. Schumaker, "On Lg-splines", J. Approx. Theory 2 (1969) 29-49.

[34] J. W. Jerome and R. S. Varga, "Generalizations of spline functions and applications to nonlinear boundary value and eigenvalue problems", in Theory and Applications of Spline Functions (ed. T. N. E. Greville), (Academic Press, New York, 1969) 103-155.

[35] J.-L. Lagrange, "Memoire sur la méthode d'interpolation", in Oeuvres de Lagrange (ed. J.-A. Serret), Volume 5, (Gauthiers-Villars, Paris, 1870) 663-684.

[36] P.-J. Laurent, Approximation et Optimisation (Hermann, Paris, 1972).

[37] V. I. Lebedev, An Introduction to Functional Analysis and Computational Mathematics (Birkhaüiser, Boston, 1997).

[38] T. R. Lucas, "A theory of generalized splines with applications to nonlinear boundary value problems", Ph. D. Thesis, Georgia Institute of Technology, 1970.

[39] T. R. Lucas, " $M$-splines", J. Approx. Theory 5 (1972) 1-14.

[40] D. V. Pai, "On nonlinear minimization problems and $L f$-splines. I", J. Approx. Theory 39 (1983) 228-235.

[41] M. J. D. Powell, "The theory of radial basis function approximation", in Adv. Num. Anal. Vol II (ed. W. Light), (OUP, Oxford, 1992).

[42] P. M. Prenter, Splines and Variational Methods (John Wiley and Sons, New York, 1975).

[43] C. Rabut, " $B$-splines polyharmoniques cardinales: Interpolation, quasi-interpolation, filtrage", Thèse, Université Paul Sabatier, Toulouse, 1990.

[44] R. Schaback, "Konstruktion und algebraische Eigenschaften von $M$-Spline-Interpolierenden", Numer. Math. 21 (1973) 166-180.

[45] I. J. Schoenberg, "Contributions to the problem of approximation of equidistant data by analytic functions. Parts A and B", Quart. Appl. Math. 4 (1946) 45-99, 112-141.

[46] I. J. Schoenberg, "On trigonometric spline interpolation", J. Math. Mech. 13 (1964) 795-825.

[47] I. J. Schoenberg, "On the Ahlberg-Nilson extension of spline interpolation: The $g$-splines and their optimal properties", J. Math. Anal. Appl. 21 (1968) 207-231.

[48] M. H. Schultz and R. S. Varga, "L-splines”, Numer. Math. 10 (1967) 345-369.

[49] G. S. Sidhu and H. L. Weinert, "Vector-valued $L g$-splines. I. Interpolating splines", J. Math. Anal. Appl. 70 (1979) 505-529.

[50] H. J. Taijeron, A. G. Gibson and C. Chandler, "Spline interpolation and smoothing on hyperspheres", SIAM J. Sci. Comput. 15 (1994) 1111-1125.

[51] J. Thomann, "Détermination et construction de fonctions Spline à deux variables définies sur un domaine rectangulaire ou circulaire", Thèse, Université de Lille, 1970.

[52] F. I. Utreras, "Convergence rates for constrained spline functions", Rev. Mat. Apl. 9 (1987) 87-95.

[53] R. S. Varga, Functional Analysis and Approximation Theory (SIAM, Philadelphia, 1971).

[54] G. Wahba, "Spline interpolation and smoothing on the sphere", SIAM J. Sci. Stat. Comp. 2 (1981) 5-16.

[55] G. Wahba, Spline Models for Observational Data (SIAM, Philadelphia, 1990).

[56] H. L. Weinert, U. B. Sesai and G. S. Sidhu, "Arma splines, system inverses, and least-squares estimates", SIAM J. Control Optim. 17 (1979) 525-536. 